\title{
Safe Driving App for Smart Phones
}

\author{
Tanvi Kamat \\ Bachelor of \\ Engineering \\ Xavier Institute of \\ Engineering \\ I.T Department
}

\author{
Sukanya \\ Narvekar \\ Bachelor of \\ Engineering \\ Xavier Institute of \\ Engineering \\ I.T Department
}

\author{
Namita Pai \\ Bachelor of \\ Engineering \\ Xavier Institute of \\ Engineering \\ I.T Department
}

\author{
Jyotsna More \\ Assistant Professor \\ Xavier Institute of \\ Engineering \\ I.T Department
}

\begin{abstract}
Experimental automobiles in the past have included sensors to record data preceding test crashes. Later after analysis, crash scenarios are analysed and studied to avoid future crashes. With more than 10 million car accidents reported in the India each year, car manufacturers have shifted their focus of a passive approach, e.g., airbags, seat belts, and antilock brakes, to something more active by adding features associated with advanced driver-assistance systems (ADASs) and collision avoidance systems. But the vehicles consisting these add-ons are extremely expensive.
\end{abstract}

Since, add-ons ultimately add on to the cost of the vehicle it is not very convenient for the user. Therefore to solve this problem we present a Smart Phone application as cell phones are already available in abundance and are also portable. Given its accessibility and portability, the smart phone application can bring a driver to assist any vehicle without regard for on-vehicle communication system requirements. Road quality tends to be variable, with bumpy roads and potholes being common place even in the heart of cities. The application Safe Driving App for Smart Phones detects these bumps and assists the driver for a safe driving. Also, if the driver is not well or if he falls asleep behind the wheel his life may be in danger. Hence an application that can detect patterns like weaving pattern and sudden acceleration and sudden deceleration is developed. That is, basically if the driver is driving at normal speed but suddenly accelerates or decelerates. To assure that the driver is safe, a message is sent along with the current location to the contacts registered by the user. Also, if the driver is perfectly fine he can stop the sending of message by touching the screen in the time window. It is an affordable and smart choice over expensive add-ons.

\section{General Terms}

Safe driving, smart phones, assist driver

\section{Keywords}

Weaving, bumps, Sudden acceleration, Sudden deceleration.

\section{INTRODUCTION}

A report of Ministry of Road Transport \& Highways, Government of India says during the year 2011, there were around 4.98 lakh road accidents, which killed 1.42 lakh people and injured more than 5 lakh persons; many of them are disabled for rest of their lives [4]. Day by day the risk to drive a vehicle has risen highly. The numbers of accidents are increasing every day. A major cause of road accidents is rash driving. Hence a system is proposed which will very efficiently alert the driver who is driving rashly. Identification of risk and taking necessary action to minimize risk is a challenge in the current technology. As of now time demands a real time monitoring and alerting system that will minimize such driving risks with fast response time.

After analysis, crash scenarios are stored and analysed with realtime driving data to potentially recognize a future crash and actually prevent it. As mentioned earlier, there are a lot of addons in the market like, e.g. ADA's, lane departure systems, etc. However, vehicles manufactured with these sensors are hard to find in lower priced economical vehicles as ADA's packages are not cheap add-ons. In addition, not all vehicles have these type of safety feature. They may only have passive safety features since manufacturers have recently begun to introduce an effective driver assist. As sensors ultimately add onto the cost of a vehicle initially and cannot be affordably upgraded, a mobile smart phone can be used as an alternative device for ADASs that can assist the driver and compliment any existing active safety features. Given its accessibility and portability, the smart phone can bring a driver assist to any vehicle without regard for on vehicle communication system requirements. As a self-contained device, mobile phone presents a mature hardware and software environment for safe driving.

The system based on smart phone can function effectively on its own because smart phones are highly portable; all necessary components are already integrated therein. The minimum requirement for such a mobile phone platform is the presence of simple sensors, e.g., accelerometer and orientation sensor. Currently, many phones, especially smart phones, meet this requirement. They contain multiple types of sensors, including accelerometers and orientation sensors [2]. And along with these sensors, because of good telecom companies the cell phone reception is also acceptable. A study says, over 120 million smart phones were sold in 2008 , and their popularity is projected to continuously increase in the near future due to their decreasing price. Recently, several leading telecommunication companies such as AT\&T have made available affordable smart phones, whose features are similar to those of high- end models, in addition to cheaper service plans.

With this as the motivation, a cheap and convenient mobile device that is able to analyse and advice the driver on sudden and harmful situations that arise from vehicle movements and environmental factors is used. This application is a type of driver assist. Providing constructive feedback to the driver is crucial in correcting bad driving behaviours. Recently, Ford and BMW have proposed ideas on this type of driver assist, where it can be integrated into their telematics system, along with hundreds of other vehicles sensors. Given the sensing capability of smart phones, internal accelerometer and GPS of the phone is used in place of the expensive hardware installed in vehicles to assist active features for driving. The Accelerometer Sensor (predicts $\mathrm{X}, \mathrm{Y}, \mathrm{Z}$ movement) is used to detect the driving moment like 
whether the driver is going faster or slower and based on this information it will draw a graph. The GPS detects the current location of the driver and sends it to the registered contacts.

\section{PROBLEM DEFINITION}

Vehicle manufacturers continue to increase their emphasis on safety with advanced driver-assistance systems

(ADASs) which include the drawbacks as 1) drivers' shifted attention to road environment information that causes insufficient attention to the primary driving tasks 2) inappropriate driver reactions (e.g. harsh braking) that results in unexpected warnings 3) driver frustration with warning Systems due to unnecessary frequent system warnings, 4) driver frustration when certain elements of the driving tasks are taken over by the system in contrast to driver's desire [3].

So a mobile phone application which advises on the safety conditions in terms of safety alerts is proposed. Mobile smart phones have inbuilt sensors which are useful while driving .The three-axis accelerometer of an Android-based smart phone is used to record and analyse various driver behaviours that could result in accidents. Along with this, if the driver gets stuck at a location, the system will help him to get the number of the nearby mechanic in that area. An application installed on the mobile phone computes accelerations based on sensor readings, and compares them with typical driving patterns for user safety. The entire solution requires only a smart phone placed in vehicle with an accelerometer and orientation sensor.

\section{SCOPE}

There is a need of a technology by which the user can get safety alerts and can get assistance from his/her relatives or friends in case of emergencies. Car accidents cannot be completely prevented but by analysing the abnormal driving patterns, user can do preventive actions to get assistance. Many car companies are trying to provide the in-built safety options but while looking at present situation these options are not sufficient. Objective of this project is to overcome these situations where there are maximum chances of accident by finding out the car's driving pattern. There will be differences between phone's orientation and vehicle's orientation. Therefore the driving behaviour detection based on vehicle accelerations might be affected. Phone location and orientation inside the car should be configured to achieve accurate measurements. Likewise, driving behaviours vary from driver to driver. A mobile Smartphone contains GPS and an accelerometer offering flexibility in methodology and user implementation. The purpose of this system is to identify the danger zones and giving audio alert through a popup with vibration to the driver for reducing speed of vehicle. This is very useful to the driver for identifying danger zones and directions and also for enhancing safety. Encouraging results in identifying numerous road anomalies and sudden driving manoeuvres allow for the system to evaluate an entire road's condition and help advice drivers on unsafe characteristics, respectively, both of which are distinguishable factors that can determine safety on the road. This sensor distinguishes the bumpy roads from the smooth ones.

\section{PROPOSED SYSTEM}

An algorithm based on accelerations, is applied to the mobile phones equipped with accelerometer and orientation sensor. The acceleration readings are usually provided by accelerometer in directions of $\mathrm{x}-, \mathrm{y}^{-}$, and $\mathrm{z}$-axis. For generality, it is assumed that the directions of $\mathrm{x}^{-}, \mathrm{y}-$, and $\mathrm{z}$-axis are decided by the orientation of the phone. As illustrated in Figure 3.1, the $\mathrm{x}$-axis has positive direction toward the right side of the device; the $y$-axis has positive direction toward the top of the device and the $\mathrm{z}$-axis has positive direction towards the front of the device. These values can be obtained via the orientation sensor. Longitudinal and lateral acceleration values are separately calculated and the result is matched with a predefined pattern to identify the abnormal driving patterns

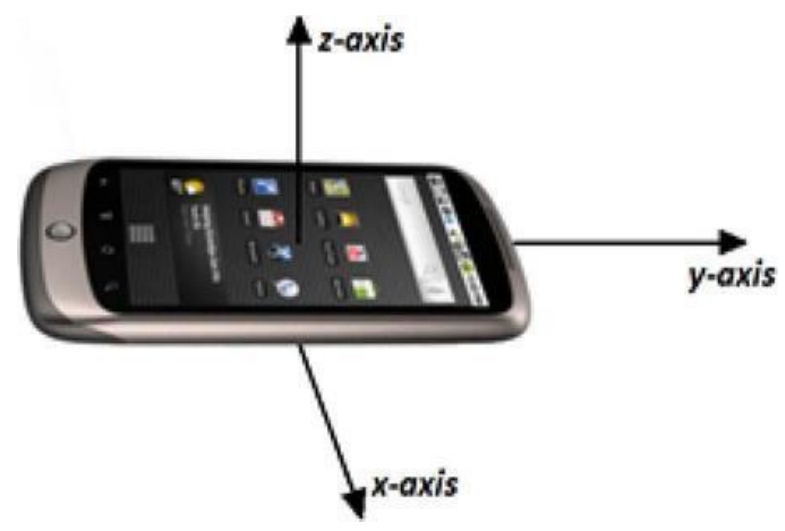

Fig.1: Three-axis diagram of the accelerometer

\subsection{Bump Detection/Crash}

Here the system considers the problem of detecting a pothole or bump on the road. A bump could arise due to a variety of reasons, both unintended (e.g., potholes), and intended (e.g., speed bumps to slow vehicles down) [6]. There is no difference between these two causes here as an external database of the intended bumps could be used to post-process the bump event when detected according to Nericell[3] to filter the ones due to intended causes.

\subsection{Sudden Acceleration and Deceleration}

Gradual increase and decrease in the acceleration will result in sudden acceleration and deceleration respectively. A slope and a maximum threshold, and it is compared with more extreme scenarios which shows a situation in which the driver quickly accelerates from rest and decelerates to a stop. Both are represented with a steep slope and short time frame. Comparison of the two will help to distinguish unsafe acceleration and deceleration pattern.

\subsection{Weaving Pattern}

This is the third condition which is to be detected. If movement of the vehicle changes continuously along the $\mathrm{x}$-axis that is if the vehicle starts moving to extreme left then extreme right frequently along the road then the motion is undesirable thus abnormal weaving pattern is detected [2].

\section{ALGORITHM FOR DETECTING DRIVINGPATTERNS}

The detection algorithm based on acceleration and it is applied to the mobile phone equipped with accelerometer and orientation sensor. The acceleration readings are usually provided by accelerometers in directions of $\mathrm{x}^{-}, \mathrm{y}-$, and $\mathrm{z}$-axis. For generality, it is assumed that the directions of $\mathrm{x}-, \mathrm{y}-$, and $\mathrm{z}$-axis are decided by the orientation of the phone. The $\mathrm{x}$-axis has positive direction towards the right side of the device, the $y$-axis has positive direction towards the top of the device and the $\mathrm{z}$-axis has positive direction towards the front of the device. Represent the rotation around $\mathrm{x}$-axis and $\mathrm{y}$-axis. In real detection process, , both the 
lateral acceleration and the longitudinal acceleration should be based on the vehicle movement direction. The acceleration information of the mobile phone should be transformed into the accelerations of the vehicle. In the simplest case, it is assumed that the mobile phone is laid flat in the vehicle, with the top of phone toward the head of vehicle, so that the accelerations on $\mathrm{x}$-axis and $\mathrm{y}$-axis represent the lateral and longitudinal accelerations of vehicle, respectively. However, the real situations are more complex.

As soon as the vehicle starts moving, its position keeps on changing. Changes in $\mathrm{x}, \mathrm{y}, \mathrm{z}$ values are displayed on the screen. A threshold is set for comparison of these values so as to detect the three above mentioned driving patterns.

When the application is launched into mobile phone, the user is supposed to add 3 contacts and it also has an option of update.

Also, a pop up message can be set in advance so that as soon as the abnormal patterns are detected, user gets a pop up message along with vibration.

The alert that is displayed on screen has two options to check confirmation of whether to send message to registered contacts or not. If the user is safe then he or she will not send the message unnecessarily. Hence the user will click "don't send" button else the user will click "send" button. Also, if the vehicle is stuck somewhere, user can get help of nearby mechanic.

The calibration procedure begins to work when the system detects the vehicle begins to move. Its starting movement gives the mobile phone a continuously initial longitudinal acceleration, either forward (to get off directly) or backward (to back off the vehicle first). It is much different from that of the human movement.

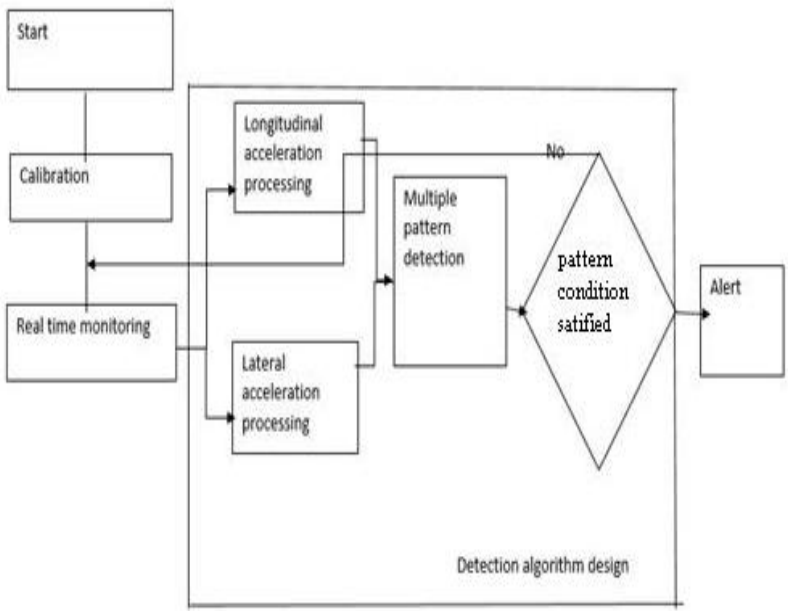

Fig.2: Working procedure of unsafe driving detection system.

The changes in $\mathrm{x}, \mathrm{y}, \mathrm{z}$ values are represented by deltax, deltay, deltaz respectively. These values are provided by the accelerometer sensor which does not require any additional hardware.

The obtained readings are compared with that of the threshold values represented by deltaxmax, deltaymax and deltazmax respectively. Once the pattern is detected, pop up is displayed. A threshold is set for vibration as well. If the acceleration is above threshold then the phone vibrates.

\section{RESULT}

Module 1: Messaging Module

Input: Contact name

Contact phone number

Auto Reply message text

Output: Auto Reply message sent if condition detected

\begin{tabular}{|c|c|}
\hline \multicolumn{2}{|l|}{ AddContact } \\
\hline \multicolumn{2}{|l|}{ Contact 1} \\
\hline \multicolumn{2}{|l|}{ Namita } \\
\hline \multicolumn{2}{|l|}{9769712110} \\
\hline & Update \\
\hline \multirow{2}{*}{\multicolumn{2}{|c|}{$\begin{array}{r}\text { Contact } 2 \\
\text { sukanya }\end{array}$}} \\
\hline & \\
\hline \multicolumn{2}{|l|}{9619850793} \\
\hline & Update \\
\hline \multicolumn{2}{|l|}{ Contact 3} \\
\hline \multicolumn{2}{|l|}{ Name } \\
\hline \multicolumn{2}{|l|}{ Number } \\
\hline & Add \\
\hline
\end{tabular}

\section{$\triangleleft \quad \bigcirc \quad \square$}

Fig 3:Add contacts page

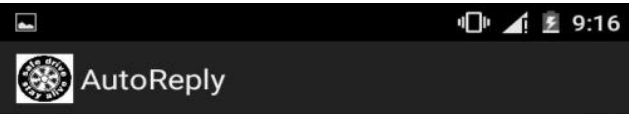

Enter your message

I'm in danger

Update

Message Saved Successfully

$\triangleleft$

○

$\square$

Fig. 4:Auto reply message page 


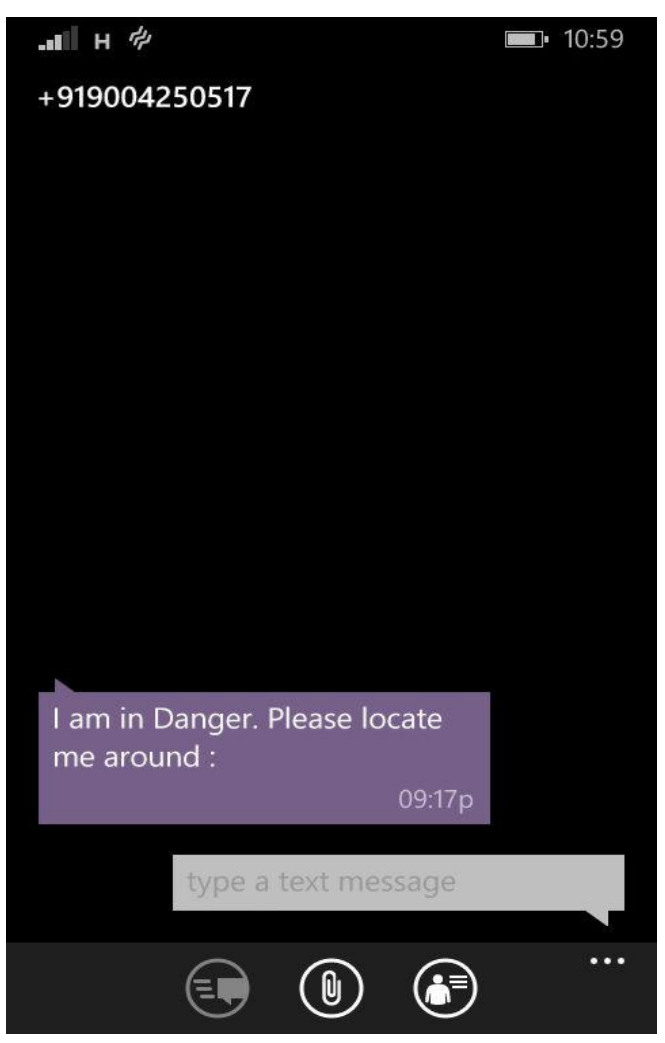

Fig. 5: Message sent to registered contact

Module 2: Bump and weaving condition detection.

The first condition is bump detection wherein a threshold is set and if the threshold is crossed a pop up is displayed and the user is given a window of 10 seconds to interact with the system.

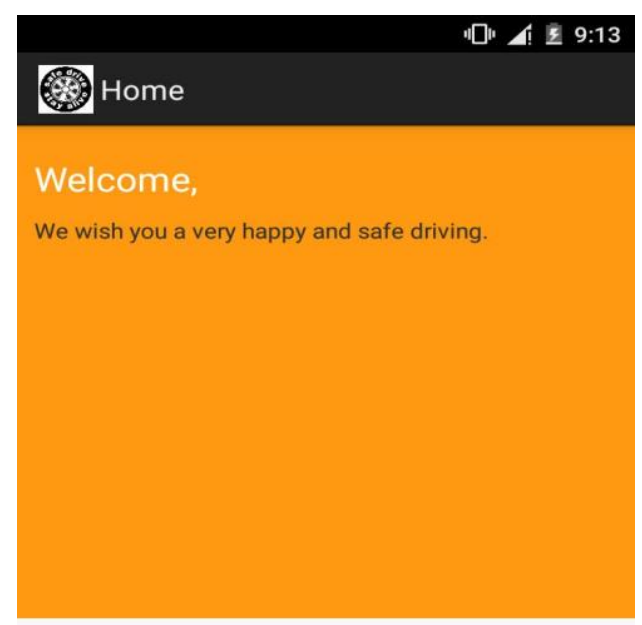

Weaving pattern detected.

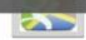

$\triangleleft$

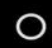

$\square$

Fig. 6: weaving pattern detected

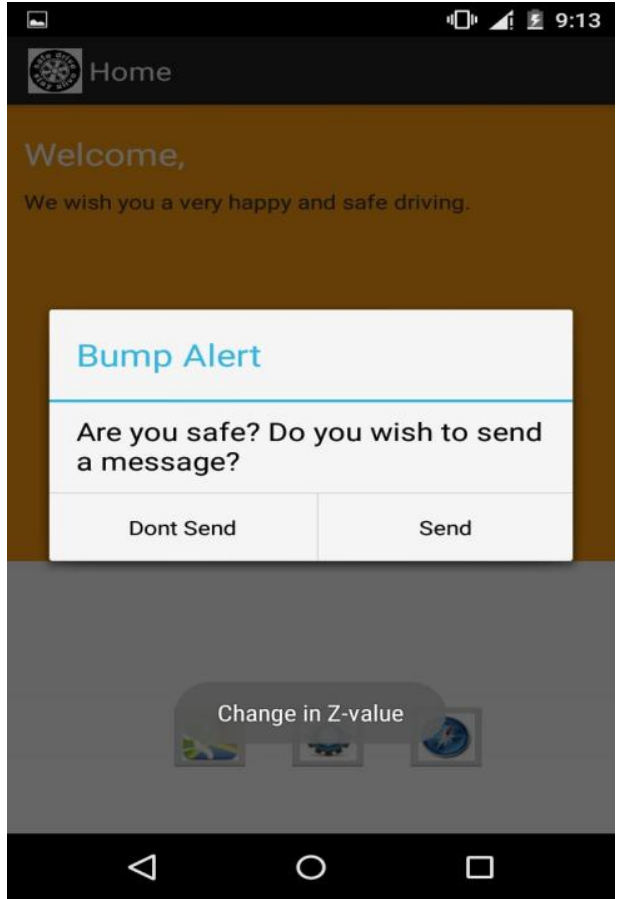

Fig. 7: Bump alert popup

\section{CONCLUSION}

By using a mobile smart phone, some innovative applications are integrated inside an automobile to evaluate a vehicle's condition, such as gear shifts, driving patterns (weaving), crashes, etc. In times of emergency when user will require contact number of the mechanic in a particular area if his car has some technical problem then there is a functionality which will help user to find number of nearby mechanic.

Along with these findings, an analysis of a driver behaviour for safe and sudden maneuvers, such as vehicle accelerations and lane changes, will be identified, which can advise drivers who are unaware of the risks they are potentially creating for themselves and neighbouring vehicles. Using GPS the last location of the user will be auto-sent to the registered VIP contacts

\section{FUTURE WORK}

The system that is being currently being developed is for detecting abnormal driving patterns to avoid future accidents. The system can be further enhanced by adding more functionalities like notification to the hospital and location based services

\subsection{Notification to Hospital}

This feature will send message to nearby hospital found with the help of GPS tracking. The message will include location of vehicle, blood group which will help to ambulance on the specified location.

\subsection{Location based Services}

Location Based Service (LBS) LBS is mobile service that has the capability to provide real time information based on the user's location.

\section{REFERENCES}

[1] Fazeen M; Gozick B; Dantu R; Bhukiya M; González M; "Safe driving using mobile Phones" IEEE Journal, Page(s): $1-7$. 
[2] Jiangpeng Dai; JinTeng; Xiaole Bai; Zhaohui Shen; Dong Xuan; "Mobile based drunk driving detection" IEEE Journal, Page(s):1-8, 2010.

[3] Prashanth Mohan; Venkata N. Padmanabhan; Ramachandran Ramjee; “.Nericell: Rich Monitoring of Road and Traffic Conditions using Mobile Smart phones" SenSys Proceedings of the 6th ACM conference on Embedded network sensorSystems, Page(s):1-14, 2008.

[4] Tanmoy D. Goswami;Shrinivas R. Zanwar;Zafar Ul. Hasan; "Android Based Rush and Drunk Driver Alerting System”In International Journal of Engineering
Research and applications, Page(s):1-4, 2014,

[5] Ming Liu, "A Study of Mobile Sensing Using Smartphones.",International Journal of Distributed Sensor Networks, Page(s):1-9,2013

[6] Ching-Yao Chan, "On the Detection of Vehicular Crashes - System Characteristics And Architecture." IEEE Journal,Vol 51, Page(s):180-194,2002.

[7] Applications of Android. Available at: https://www.istos.ws/index.php/services/mobileapplication- services/android-application 